Published in final edited form as:

Mov Disord. 2013 September ; 28(10): 1384-1390. doi:10.1002/mds.25575.

\title{
Rivastigmine is Associated with Restoration of Left Frontal Brain Activity in Parkinson's Disease
}

\author{
Katherine L. Possin, PhD ${ }^{1}$, Gail A. Kang, MD ${ }^{1}$, Christine Guo, $\mathrm{PhD}^{1}$, Eric M. Fine, PhD ${ }^{1}$, \\ Andrew J. Trujillo, BS ${ }^{1}$, Caroline A. Racine, PhD $^{1}$, Reva Wilheim, BS ${ }^{1}$, Erica T. Johnson, \\ BS $^{1}$, Jennifer L. Witt, MD ${ }^{1}$, William W. Seeley, MD ${ }^{1}$, Bruce L. Miller, MD ${ }^{1}$, and Joel H. \\ Kramer, PsyD ${ }^{1}$ \\ ${ }^{1}$ University of California, San Francisco, Department of Neurology
}

\begin{abstract}
Objective-To investigate how acetylcholinesterase inhibitor (ChEI) treatment impacts brain function in Parkinson's disease (PD).

Methods-Twelve patients with PD and either dementia or mild cognitive impairment underwent task-free functional magnetic resonance imaging before and after three months of ChEI treatment and were compared to 15 age and sex matched neurologically healthy controls. Regional spontaneous brain activity was measured using the fractional amplitude of low frequency fluctuations.
\end{abstract}

675 Nelson Rising Lane, Ste 190, MC 1207, San Francisco, CA 94158, Tel: 415/476-1889, Fax: 415/476-4800, kpossin@memory.ucsf.edu.

Author Roles and Financial Disclosures

Possin: Led the design and execution of analyses and writing of the manuscript. Dr. Possin has received support from K23AG037566, the Hellman Family Foundation, and the Michael J. Fox Foundation. She is employed by UCSF.

Kang: Organization and execution of research project and review of manuscript. Dr. Kang is employed in private practice.

Guo: Assisted with the design and execution of analyses and review of manuscript. During the last year, Dr. Guo was employed by UCSF. She reports no disclosures.

Fine: Execution of research project and analyses and review of manuscript. Dr. Fine is employed by UCSF and by Stanford University. He reports no disclosures.

Trujillo: Assisted with the design and execution of analyses and review of manuscript. Mr. Trujillo is employed by UCSF. He reports no disclosures.

Racine: Conception of research project and review of manuscript. Dr. Racine is employed by UCSF and receives funding from the North American Gamma Knife Consortium (P0053752).

Wilheim: Organization and execution of research project. Ms. Wilheim has been employed by UCSF and is currently a student at the University of Arizona, Tucson. She reports no disclosures.

Johnson: Assisted with the execution of analyses and review of manuscript. Ms. Johnson is employed by UCSF. She reports no disclosures.

Witt: Assisted with the organization and execution of the research project. She currently works in private practice. She reports no disclosures.

Seeley: Conception, organization, and execution of research project; design of analyses; and review of manuscript. Dr. Seeley is a consultant for Summer Street Research Partners, is on the advisory board of Bristol-Myers Squibb, and receives grant support from the National Institute on Aging, the Tau Consortium, the Alzheimer's Disease Drug Foundation - Association for Frontotemporal Dementia, and the James S. McDonnell Foundation.

Miller: Conception of project and review of manuscript. Dr. Miller receives grant support from the National Institute on Aging and serves as a consultant for TauRx, Allon Therapeutics, Lilly USA LLC, and Seimens Medical Solutions. He is on the Board of Directors for the John Douglas French Foundation for Alzheimer's Research and for The Larry L. Hillblom Foundation. Kramer: Conception, organization, and execution of research project; design of analyses; and review of manuscript. Joel Kramer receives research support from NIH grants P50 AG023501 R01AG022983 and R01AG032289. He receives honoraria for serving on the University of Indiana Alzheimers Disease Center external advisory board. He receives royalties from the California Verbal Learning Test. He previously received support from Novartis Pharmaceuticals to conduct the present study. 
Results-At baseline, patients showed reduced spontaneous brain activity in regions important for motor control (e.g., caudate, supplementary motor area, precentral gyrus, thalamus), attention and executive functions (e.g., lateral prefrontal cortex), and episodic memory (e.g., precuneus, angular gyrus, hippocampus). After treatment, the patients showed a similar but less extensive pattern of reduced spontaneous brain activity relative to controls. Spontaneous brain activity deficits in the left premotor cortex, inferior frontal gyrus, and supplementary motor area were restored such that the activity was increased post-treatment compared to baseline and was no longer different from controls. Treatment-related increases in left premotor and inferior frontal cortex spontaneous brain activity correlated with parallel reaction time improvement on a test of controlled attention.

Conclusions-PD patients with cognitive impairment show numerous regions of decreased spontaneous brain function compared to controls, and rivastigmine is associated with performance-related normalization in left frontal cortex function.

\section{Keywords}

acetylcholine (D02.092.211.111); magnetic resonance imaging functional (E01.370.350.825.500); attention (F02.830.104.214); Acetylcholinesterase Inhibitors (D27.505.519.389.275) executive control (F02.463.217)

\section{Introduction}

Cholinergic deficits are pronounced in Parkinson's disease (PD) and are a primary substrate of debilitating cognitive symptoms including attention and executive dysfunction (1-4). Acetylcholinesterase inhibitors (ChEIs), including rivastigmine and donepezil, have been associated with symptomatic improvements $(5,6)$. In a randomized controlled study of rivastigmine in 487 patients with PDD, significant benefits over placebo were seen on four measures of attention (7). Treatment was associated with enhanced attention compared to baseline, suggesting that rivastigmine was not simply preventing further deterioration but rather improved brain activity relevant for attention.

To elucidate the neural systems that underlie the effects of ChEIs on attention in PD, we investigated spontaneous brain activity in cognitively impaired PD patients compared to controls at baseline and after 3 months of rivastigmine treatment. To measure brain activity, we selected fractional amplitude of low frequency fluctuations (fALFF), a metric derived from task-free functional magnetic resonance imaging (fMRI) that represents the power of regional spontaneous and intrinsic brain activity at the local, voxel-wise level while the subject is at rest $(8,9)$. More specifically, the amplitude of low-frequency fluctuations (ALFF) is the total power in the low-frequency range, and fALFF is calculated by dividing ALFF by the total power across all measurable frequencies. Whereas ALFF values increase near blood vessels and CSF, likely due to pulsations in those areas, fALFF is less susceptible to artifactual signals $(8,10)$. Inter-individual differences in fALFF at rest were recently shown to predict performance on an Eriksen flanker test of attention, and in addition, to predict the magnitude of BOLD activation evoked during flanker task performance during a separate fMRI scan (11). This study and others $(9,12)$ validate fALFF as a measure of intrinsic brain activity relevant to evoked brain activity and behavior. 
We hypothesized that prior to rivastigmine treatment, spontaneous brain activity would be lower in PD compared to controls, with the most notable decreases in motor areas including the striatum and motor cortex that are affected by PD, and in regions affected in the earlier stages of Lewy body deposition including the brainstem, thalamus, striatum, and mesocortex (13). The nucleus basalis - neocortical cholinergic system contributes greatly to attentional function (14) and ChEIs improve attention and executive functions in PDD $(1,7)$.

Accordingly, we hypothesized that rivastigmine would restore brain activity to normal levels in regions important for the control of attention including the frontal lobes. Further, we hypothesized that this restoration of brain activity would correlate with improvements in controlled attention. Cholinergic activity in prefrontal cortex facilitates cue detection in rats and is thought to bias attention from a default state to a state fostering vigilance toward sensory information (15-17). Guided by these studies, we selected a test of controlled attention that requires sustained vigilance and rapid cue detection.

\section{Methods}

\section{Subjects}

Patients were recruited from University of California, San Francisco (UCSF) movement disorders neurologists, regional PD support group meetings and advertisements in PD newsletters. Exclusion criteria included sinus bradycardia; abnormal Vitamin B12, methylmalonic, RPR or TSH levels (unless on treatment for thyroid condition); prior brain surgery; history of significant drug or alcohol abuse; history of encephalitis, multiple sclerosis, CNS infection, epilepsy, or primary CNS disease besides PD; prior exposure to neuroleptic agents; and use of ChEI within 6 weeks of baseline evaluation of study. Neurologically healthy controls matched on age, gender, and education were recruited from the UCSF Memory and Aging Center project on healthy aging (18) (Table 1). All subjects or their responsible family members gave written informed consent prior to participation in the study, which was approved by the UCSF committee on human research.

Diagnoses of patients and controls were based upon neurological history and examination, an interview with a family member, and a brief neuropsychological assessment previously described (19). Three patients, from an original sample of 15 , dropped out due medication side effects: lightheadedness or dizziness in 2 subjects and gastrointestinal distress in 1 subject. In the sample that completed the study, 6 patients were diagnosed as PDD (20) and 6 patients were diagnosed as PD-MCI (21). The MCI subtypes were MCI-executive ( $\mathrm{N}=1)$, MCI-executive, memory ( $\mathrm{N}=1$ ), MCI-executive, visuospatial ( $\mathrm{N}=1)$, MCI-executive, memory, attention/working memory $(\mathrm{N}=2)$, and $\mathrm{MCI}$-executive, memory, attention/working memory, visuospatial $(\mathrm{N}=1)$.

\section{Study Medication}

The Exelon patch (rivastigmine, Novartis International AG, Basel, Switzerland) was administered at a dosage of $4.6 \mathrm{mg} / 24$ hours from baseline to week 4 and at $9.5 \mathrm{mg} / 24$ hours from week 4 to 12. All patients were taking optimal doses of dopamine replacement therapy prescribed by their neurologist. No subjects initiated new medications or new dosages of current medications during the study. Neuroimaging and cognitive testing occurred at the 
same time of day at baseline and post-treatment whilst "on" to minimize the effects of dopamine fluctuations on brain activity. We did not withhold dopaminergic medications during image acquisition because head motion can compromise image quality (22).

\section{Clinical Assessments}

The Unified Parkinson's Disease Rating Motor Scale (UPDRS) was administered by a movement disorders trained neurologist at baseline after 12 hours of PD medication withholding. The cognitive assessment was performed at least 45 minutes after subjects took their PD medications when the patients reported feeling in the "on" state. Two cognitive measures were administered at baseline and post-treatment: the Montreal Cognitive Assessment Scale (MoCA) to measure global cognition, and a longer version of the NIH EXAMINER Continuous Performance Test (CPT) to measure controlled attention (examiner.ucsf.edu). On the CPT, subjects press the spacebar quickly when they see a target image (a white star; 150 trials), and withhold response when they see a non-target image (5 randomly sampled white shapes; 150 trials). The inter-stimulus interval is randomly sampled from $1.5 \mathrm{~s}, 2.5 \mathrm{~s}$, or $4 \mathrm{~s}$. Performance is measured by the median reaction time (milliseconds) on accurate target trials. Accuracy was high with only one poor performance at baseline (67\% accurate) and all other performances exceeding $88 \%$. Due to minimal variance in accuracy, we did not analyze accuracy further. Excluding the subject with poor accuracy at baseline did not alter any of the CPT results and we retained his data. Data were missing for two of the patients on the CPT post-treatment due to a computer error.

\section{Image Acquisition}

Magnetic resonance images of all subjects were acquired at the UCSF Neuroscience Imaging Center on a 3 Tesla Siemens Tim Trio scanner equipped with a 12-channel receiver head coil. A volumetric magnetization prepared rapid gradient echo (MP-RAGE) sequence was used to obtain T1-weighted images of the entire brain (TR/TE/TI $=2300 / 3 / 900 \mathrm{~ms}$, flip angle of 9 degrees, a bandwidth of $240 \mathrm{~Hz} /$ pixel, sagittal orientation with a FOV $=256 \times$ $240 \mathrm{~mm}, 1 \times 1 \times 1 \mathrm{~mm}^{3}$ resolution, and 160 slices). "Resting state" or task-free fMRI scans were obtained using 36 axial slices ( $3 \mathrm{~mm}$ thick) parallel to the plane connecting the anterior and posterior commissures and covering the whole brain using a $\mathrm{T} 2 *$-weighted gradient echo-echo planar sequence $\left(\mathrm{TR} / \mathrm{TE}=2000 / 27 \mathrm{~ms}\right.$, flip angle $80^{\circ} ; \mathrm{FOV}=230 \times 230 \mathrm{~mm}$; matrix size: $92 \times 92 ; 3 \mathrm{~mm}$ slices with $2.5 \times 2.5 \times 3 \mathrm{~mm}$ resolution). All subjects underwent 8 minutes of scanning ( 240 images) after being instructed only to remain awake with their eyes closed.

\section{Image Preprocessing}

Image processing was performed with SPM8. After discarding the first eight frames to allow for magnetic field stabilization, functional images were realigned, slice-time corrected, coregistered to their T1-weighted images, normalized to standard space and smoothed with a $4 \mathrm{~mm}$ full-width at half-maximum Gaussian kernel (normalization and smoothing were carried out in one step using DARTEL toolbox). The images were resampled at a voxel size of $2 \mathrm{~mm}^{3}$. 


\section{Head Motion Assessment}

Head movements were assessed using the rigid body parameters provided by the SPM8 realign algorithm. All subjects had minimal movements (maximum translation $<2 \mathrm{~mm}$ and maximum rotation $<2$ degrees). To assess for group differences and differences between the two time points in head motion, mean root-mean-square (RMS) values of volume-to-volume changes were computed for translation and mean Euler angles for rotation, as these summary metrics have been shown to correlate with network connectivity strength (22). At neither time point did the PD patients differ in translational or rotational movement compared to controls, nor did the two time points within the PD group differ from each other, all $p$ s > .05 (Table 1).

\section{fALFF Preprocessing and Group Analyses}

Amplitude of low frequency analysis was performed using the REST toolbox, following previous methods (8). After preprocessing, each voxel's BOLD signal time series was detrended and transformed to the frequency domain to obtain the power spectrum. The power of a given frequency is measured as the square of the amplitude at each frequency of the power spectrum. We focused on the low frequency range from $0.01-0.08 \mathrm{~Hz}$ because coherent fluctuations in this range are dominant in gray matter and are thought to reflect neural activity, whereas coherent fluctuations above this range are more influenced by cardiovascular or respiratory fluctuations (10). To further reduce the impact of physiological artifact, fALFF was computed by dividing the sum of the square roots across the 0.01-0.08 $\mathrm{Hz}$ range by that across the entire frequency range $(0-0.25 \mathrm{~Hz})$. fALFF methods have been detailed and validated in previous publications $(9-12,23-25)$.

fALFF group comparisons were evaluated using t-tests and statistically thresholded using the joint probability distribution method to correct for multiple comparisons at the whole brain level (26), $p<0.01$ for voxel height and $p<0.05$ for cluster extent. Voxel-wise fALFF in gray matter was compared between controls and patients, separately for each time point. To determine if there were treatment-associated changes in brain activity, we compared the patients at baseline to post-treatment. Because we were interested in the effects of ChEIs on abnormal activity, only regions with reduced fALFF compared to controls at either time point were included.

Two clusters differed in AALFF between baseline and post-treatment, and we computed mean fALFF of those voxels. To determine whether the changes resulted in a normalization of brain activity, we compared the mean fALFF values of the patients at each time point to controls using t-tests. In addition, to determine the behavioral relevance of these changes, mean fALFF change scores were calculated for these clusters in the patients (post-treatment minus baseline) and were entered into correlation analyses with changes in the cognitive variables (post-treatment minus baseline). 


\section{Results}

\section{Performance on the cognitive measures}

On the MoCA, the patients did not differ between baseline, $23.0+/-4.8$, and post-treatment, $24.3+/-4.7$, assessments, $p=0.16$. On the CPT, the patients' median reaction time did not differ between baseline, $579+/-108$, and post-treatment, $571+/-108$, assessments, $p=$ 0.44 .

\section{Spontaneous brain activity differences between patients and controls}

At both baseline and post-treatment, the patients exhibited reduced fALFF compared to controls in several lateral and medial frontal regions including motor areas (e.g., bilateral supplementary motor area) and regions important for executive control (bilateral superior, middle, and inferior frontal gyri). The left hippocampus, the bilateral precuneus and the bilateral angular gyrus, which are regions often targeted by Alzheimer's disease, also showed reduced fALFF at both timepoints. Regarding subcortical structures, the bilateral caudate and the left thalamus showed decreased fALFF at baseline, and the right caudate and the left thalamus showed decreased fALFF at post-treatment, compared to controls. We found prominent medial occipital reductions, particularly at baseline. Overall, a similar group-level pattern of decreased fALFF was found at both time points compared to controls, but the deficits were more extensive at baseline (4,614 voxels) than post-treatment $(2,339$ voxels) (Supplementary Tables; Figure 1). The patients did not show increased fALFF in any regions compared to controls at either time point.

\section{Normalization of spontaneous brain activity associated with treatment}

To determine whether rivastigmine had any restorative effects on brain function, we created a mask of regions where the patients showed abnormally low fALFF at either timepoint (i.e., the regions depicted in Figure 1). Next, we compared regional fALFF voxel-wise between baseline and post-treatment within the mask. Results indicated that after treatment the patients showed increased fALFF in two clusters: (1) the left precentral gyrus/inferior frontal gyrus pars opercularis and (2) the left supplementary motor area (Table 2). No regions showed higher fALFF at baseline compared to post-treatment. To determine whether these fALFF changes reflected a restoration of brain function to normal levels, mean fALFF was extracted from all voxels within each cluster for patients at each time point and for the controls. As expected, the patients showed reduced fALFF compared to controls in both the left precentral/inferior frontal gyri cluster, $d=-1.56$, and the left supplementary motor area cluster, $d=-1.50$, both $p s<0.001$. These levels were restored post-treatment such that the patients no longer differed significantly from controls in the left precentral/inferior frontal gyri, $d=-0.25, p=0.52$, or the left supplementary motor area, $d=-0.69, p=0.09$. Because the patient-only within-subjects comparison was used to identify the two clusters, the observed "normalization" was not the result of statistical dependency (27). 


\section{Correlations between post-treatment increases in fALFF and changes in cognitive performance}

To determine whether the treatment-associated increases in AALFF were behaviorallyrelevant, we correlated the increases in fALFF with changes in global cognition (MoCA) and controlled attention (CPT). Increases in fALFF in the left premotor/inferior frontal gyri cluster correlated with decreases in reaction time on the CPT, $r=-0.82, p<0.01$, but not change on the MoCA, $r=0.18, p=0.58$. In the left supplementary motor area cluster, increased fALFF did not correlate significantly with changes on the CPT, $r=-0.35, p=$ 0.36 , or the MoCA, $r=0.26, p=0.41$ (Figure 2).

\section{Discussion}

Rivastigmine is an FDA-approved medication for the symptomatic treatment of PDD with beneficial effects on cognitive and psychiatric symptoms, including attention and executive functions (5). To investigate the neural systems influenced by the treatment effect, we examined spontaneous brain activity before and after 3 months of rivastigmine treatment in a cognitively impaired PD sample versus controls using fALFF, a new measure of spontaneous brain activity intensity derived from task-free fMRI (8). At baseline, the PD patients showed numerous regions of decreased spontaneous brain activity and no regions of increased spontaneous brain activity compared to controls. After 3 months of treatment, the pattern of decreased spontaneous brain activity was topographically similar but less extensive. Rivastigmine treatment was associated with restoration of spontaneous brain activity in the left premotor gyrus, the left inferior frontal gyrus pars opercularis, and the left supplementary motor area. The degree of treatment-associated increases in left premotor and inferior frontal gyri brain activity was associated with improvements in controlled attention. The results suggest that rivastigmine may support cognitive function in cognitively impaired PD patients by enhancing left frontal lobe brain activity.

In PD and PDD, cholinergic deficits are profound and widespread (28). Although ChEIs impact a diverse range of cognitive functions in $\mathrm{PD}$, frontally-mediated and externallyfocused attention and executive functions appear to benefit the most $(1,2)$. In a previous SPECT study, ChEI treatment in PDD was associated with increases in regional cerebral blood flow in the bilateral anterior cingulate cortex, the bilateral superior frontal gyri, the left precentral gyrus, the left superior frontal gyrus, and bilateral frontal white matter (29). In the present study, increases in spontaneous brain activity in left frontal regions were associated with improvements in controlled attention. Why ChEI treatment preferentially enhances brain activity in the left hemisphere remains uncertain, but this lateralization result could help explain the strong correlation with the CPT, which was completed using the right hand in all individuals. The CPT requires successful interplay between controlled attention and motor preparation of the dominant hand. Taken together, ChEI treatment in cognitively impaired PD may help to restore brain activity in left frontal regions and support frontal functions including controlled attention.

At baseline, we detected numerous regions of decreased spontaneous brain activity in the patients compared to controls. The caudate and lateral frontal cortex findings are consistent with known frontal-striatal dysfunction in PD (30). The prominent medial occipital 
reductions may reflect the severe acetylcholine activity reductions in this region (28). Findings of decreased spontaneous brain activity in the precuneus, posterior cingulate, and the angular gyrus might reflect the co-occurrence of Alzheimer's pathology in some of the sample. Interestingly, decreased spontaneous brain activity was not observed in the brainstem or amygdala, some of the earliest areas affected by Lewy pathology (13). Our results converge with previous findings of ALFF reductions in the bilateral supplementary motor area, the left middle frontal gyrus, the right precentral gyrus, the inferior occipital gyrus and the cerebellum (31), and of ALFF reductions in the left rolandic operculum(32) in PD. The only previous report of fALFF in PD also found prefrontal decreases whilst ON but not OFF dopaminergic medication (33).

The present study lacked a placebo-treated comparison sample, and it is possible that some factor other than the effect of rivastigmine, such as disease progression, caused the functional brain changes. Post-treatment fALFF results were much more similar to controls, however, than were the pre-treatment results, and gains in left frontal regions were paralleled by improved attention. These observations suggest that a positive "restoring" treatment effect, rather than disease progression, is likely to account for the reported findings. It is also important to note that PD patients respond variably to $\operatorname{ChEIs(7),~and~not~}$ all patients in this study evidenced improved attention. Those that responded in terms of improved attention were more likely to show the increased frontal brain activity.

In summary, we used a new metric of brain activity measured during task-free fMRI to evaluate the effects of rivastigmine in cognitively impaired PD. This metric appears to be sensitive to the pathophysiological effects of $\mathrm{PD}$, as the patients showed extensive reductions in spontaneous brain activity compared to controls. Treatment was associated with a restoration of spontaneous brain activity in the left precentral and inferior frontal gyri and in the left supplementary motor area. Brain activity increase in the left precentral and inferior frontal gyri was associated with improvements in controlled attention but not in global cognitive function. ChEI treatment may improve attention and executive functions by increasing the power of spontaneous brain activity in the left frontal lobe.

\section{Supplementary Material}

Refer to Web version on PubMed Central for supplementary material.

\section{Acknowledgments}

This study was supported by grants from Novartis Pharmaceuticals (Kramer, CENA713D US45T), the National Institute on Aging (Possin, K23AG037566), and by the Larry L. Hillblom Foundation (Miller, 2007/2I). We are grateful to our research participants for their generous time and efforts.

\section{References}

1. Bohnen NI, Kaufer DI, Hendrickson R, et al. Cognitive correlates of cortical cholinergic denervation in Parkinson's disease and parkinsonian dementia. J Neurol. 2006; 253(2):242-247. [PubMed: 16133720]

2. Bohnen NI, Albin RL. The cholinergic system and Parkinson disease. Behav Brain Res. 2011; 221(2):564-573. [PubMed: 20060022] 
3. Emre M, Cummings JL, Lane RM. Rivastigmine in dementia associated with Parkinson's disease and Alzheimer's disease: similarities and differences. J Alzheimers Dis. 2007; 11(4):509-519. [PubMed: 17656830]

4. Bohnen NI, Kaufer DI, Ivanco LS, et al. Cortical cholinergic function is more severely affected in parkinsonian dementia than in Alzheimer disease: an in vivo positron emission tomographic study. Arch Neurol. 2003; 60(12):1745-1748. [PubMed: 14676050]

5. Emre M, Aarsland D, Albanese A, et al. Rivastigmine for dementia associated with Parkinson's disease. N Engl J Med. 2004; 351(24):2509-2518. [PubMed: 15590953]

6. Weintraub D, Somogyi M, Meng X. Rivastigmine in Alzheimer's Disease and Parkinson's Disease Dementia: An ADAS-Cog Factor Analysis. Am J Alzheimers Dis Other Demen.

7. Wesnes KA, McKeith I, Edgar C, Emre M, Lane R. Benefits of rivastigmine on attention in dementia associated with Parkinson disease. Neurology. 2005; 65(10):1654-1656. [PubMed: 16301500]

8. Zou QH, Zhu CZ, Yang Y, et al. An improved approach to detection of amplitude of low-frequency fluctuation (ALFF) for resting-state fMRI: fractional ALFF. J Neurosci Methods. 2008; 172(1): 137-141. [PubMed: 18501969]

9. Guo, C.; Gorno-Tempini, ML.; Gesierich, B., et al. Multimodal connectivity to the anterior temporal hub: an integrated account of semantic memory. under review

10. Zuo XN, Di Martino A, Kelly C, et al. The oscillating brain: complex and reliable. Neuroimage. 2010; 49(2):1432-1445. [PubMed: 19782143]

11. Mennes M, Zuo XN, Kelly C, et al. Linking inter-individual differences in neural activation and behavior to intrinsic brain dynamics. Neuroimage. 2012; 54(4):2950-2959. [PubMed: 20974260]

12. Hoptman MJ, Zuo XN, Butler PD, et al. Amplitude of low-frequency oscillations in schizophrenia: a resting state fMRI study. Schizophr Res. 2010; 117(1):13-20. [PubMed: 19854028]

13. Braak H, Ghebremedhin E, Rub U, Bratzke H, Del Tredici K. Stages in the development of Parkinson's disease-related pathology. Cell Tissue Res. 2004; 318(1):121-134. [PubMed: 15338272]

14. Everitt BJ, Robbins TW. Central cholinergic systems and cognition. Annu Rev Psychol. 1997; 48:649-684. [PubMed: 9046571]

15. Parikh V, Kozak R, Martinez V, Sarter M. Prefrontal acetylcholine release controls cue detection on multiple timescales. Neuron. 2007; 56(1):141-154. [PubMed: 17920021]

16. Hasselmo ME, Sarter M. Modes and models of forebrain cholinergic neuromodulation of cognition. Neuropsychopharmacology. 2011; 36(1):52-73. [PubMed: 20668433]

17. McGaughy J, Kaiser T, Sarter M. Behavioral vigilance following infusions of 192 IgG-saporin into the basal forebrain: selectivity of the behavioral impairment and relation to cortical AChE-positive fiber density. Behav Neurosci. 1996; 110(2):247-265. [PubMed: 8731052]

18. Bettcher BM, Wilheim R, Rigby T, et al. C-reactive protein is related to memory and medial temporal brain volume in older adults. Brain Behav Immun. 26(1):103-108. [PubMed: 21843630]

19. Kramer JH, Jurik J, Sha SJ, et al. Distinctive neuropsychological patterns in frontotemporal dementia, semantic dementia, and Alzheimer disease. Cogn Behav Neurol. 2003; 16(4):211-218. [PubMed: 14665820]

20. Dubois B, Burn D, Goetz C, et al. Diagnostic procedures for Parkinson's disease dementia: recommendations from the movement disorder society task force. Mov Disord. 2007; 22(16): 2314-2324. [PubMed: 18098298]

21. Litvan I, Aarsland D, Adler CH, et al. MDS Task Force on mild cognitive impairment in Parkinson's disease: critical review of PD-MCI. Mov Disord. 2011; 26(10):1814-1824. [PubMed: 21661055]

22. Van Dijk KR, Sabuncu MR, Buckner RL. The influence of head motion on intrinsic functional connectivity MRI. Neuroimage. 2012; 59(1):431-438. [PubMed: 21810475]

23. Zang YF, He Y, Zhu CZ, et al. Altered baseline brain activity in children with ADHD revealed by resting-state functional MRI. Brain Dev. 2007; 29(2):83-91. [PubMed: 16919409]

24. Wang Z, Yan C, Zhao C, et al. Spatial patterns of intrinsic brain activity in mild cognitive impairment and Alzheimer's disease: a resting-state functional MRI study. Hum Brain Mapp. 32(10):1720-1740. [PubMed: 21077137] 
25. Flodin P, Gospic K, Petrovic P, Fransson P. Effects of L-dopa and oxazepam on resting-state functional magnetic resonance imaging connectivity: a randomized, cross-sectional placebo study. Brain Connect. 2012; 2(5):246-253. [PubMed: 22957904]

26. Poline JB, Worsley KJ, Evans AC, Friston KJ. Combining spatial extent and peak intensity to test for activations in functional imaging. Neuroimage. 1997; 5(2):83-96. [PubMed: 9345540]

27. Kriegeskorte N, Simmons WK, Bellgowan PS, Baker CI. Circular analysis in systems neuroscience: the dangers of double dipping. Nat Neurosci. 2009; 12(5):535-540. [PubMed: 19396166]

28. Shimada H, Hirano S, Shinotoh H, et al. Mapping of brain acetylcholinesterase alterations in Lewy body disease by PET. Neurology. 2009; 73(4):273-278. [PubMed: 19474411]

29. Ceravolo R, Volterrani D, Frosini D, et al. Brain perfusion effects of cholinesterase inhibitors in Parkinson's disease with dementia. J Neural Transm. 2006; 113(11):1787-1790. [PubMed: 16758132]

30. Sawamoto N, Piccini P, Hotton G, Pavese N, Thielemans K, Brooks DJ. Cognitive deficits and striato-frontal dopamine release in Parkinson's disease. Brain. 2008; 131(Pt 5):1294-1302. [PubMed: 18362097]

31. Skidmore FM, Yang M, Baxter L, et al. Reliability analysis of the resting state can sensitively and specifically identify the presence of Parkinson disease. Neuroimage. 2011

32. Long D, Wang J, Xuan M, et al. Automatic classification of early Parkinson's disease with multimodal MR imaging. PLoS One. 2012; 7(11):e47714. [PubMed: 23152757]

33. Kwak Y, Peltier SJ, Bohnen NI, Muller ML, Dayalu P, Seidler RD. L-DOPA changes spontaneous low-frequency BOLD signal oscillations in Parkinson's disease: a resting state fMRI study. Front Syst Neurosci. 2012; 6:52. [PubMed: 22783172]

34. Tomlinson CL, Stowe R, Patel S, Rick C, Gray R, Clarke CE. Systematic review of levodopa dose equivalency reporting in Parkinson's disease. Mov Disord. 25(15):2649-2653. [PubMed: 21069833] 


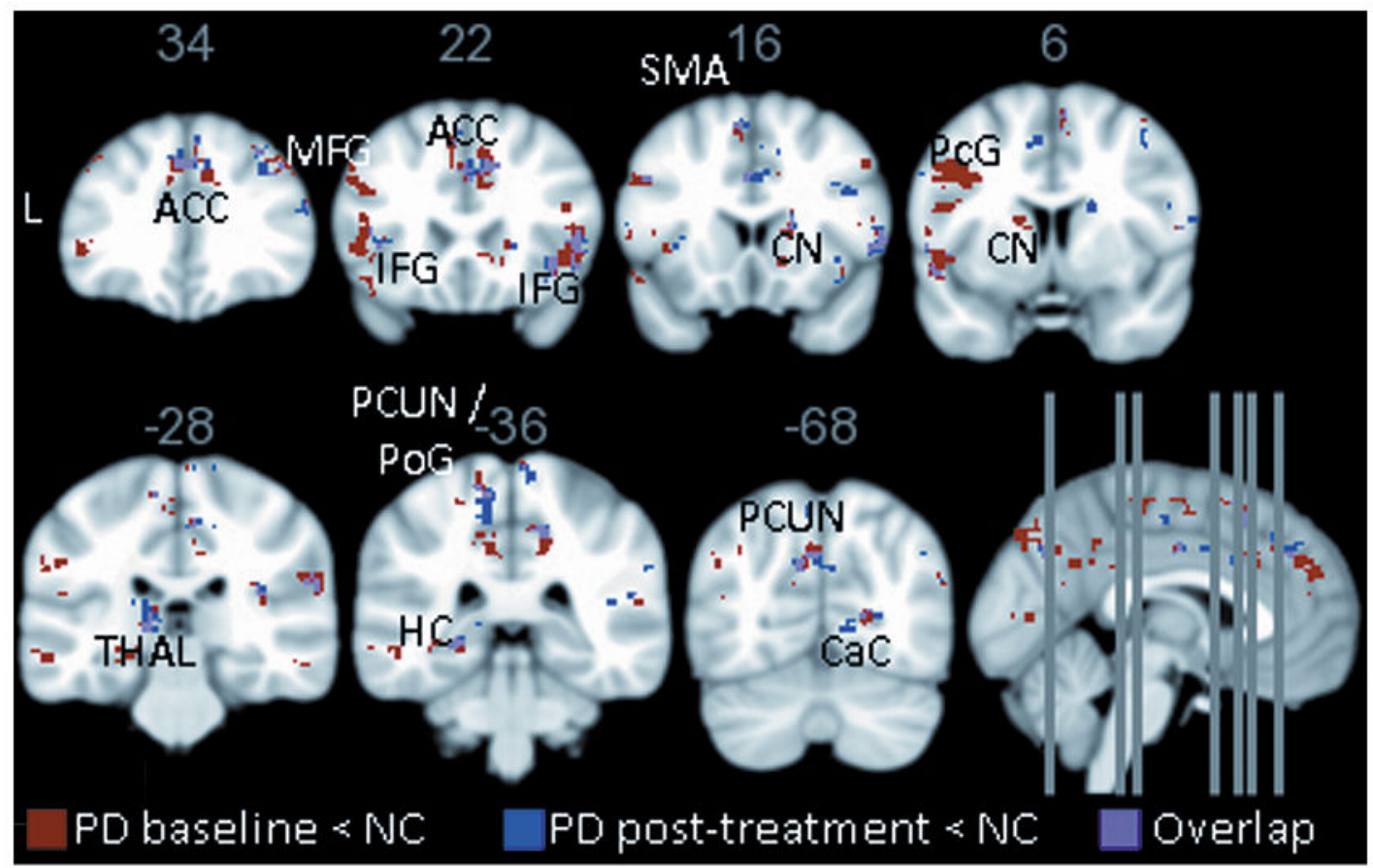

Figure 1.

Patients with PD show distributed reductions compared to controls in brain activity as measured by fALFF at both baseline (red) and post-treatment (blue); the overlap is shown in purple. Results are generated with a joint height-extent threshold ( $\mathrm{p}<0.01$ peak height and $\mathrm{p}<0.05$ extent). PD showed no significant increases compared to controls at either timepoint. $\mathrm{ACC}=$ anterior cingulate; $\mathrm{MFG}=$ middle frontal gyrus; $\mathrm{IFG}=$ inferior frontal gyrus; $\mathrm{SMA}=$ supplementary motor area; $\mathrm{CN}=$ caudate nucleus; $\mathrm{PcG}=$ precentral gyrus; $\mathrm{THAL}=$ thalamus $; \mathrm{PCUN}=$ precuneus $; \mathrm{PoG}=$ postcentral gyrus $; \mathrm{HC}=$ hippocampus $; \mathrm{CaC}=$ calcarine cortex; $\mathrm{PD}=$ Parkinson's disease NC = normal controls. 
A.
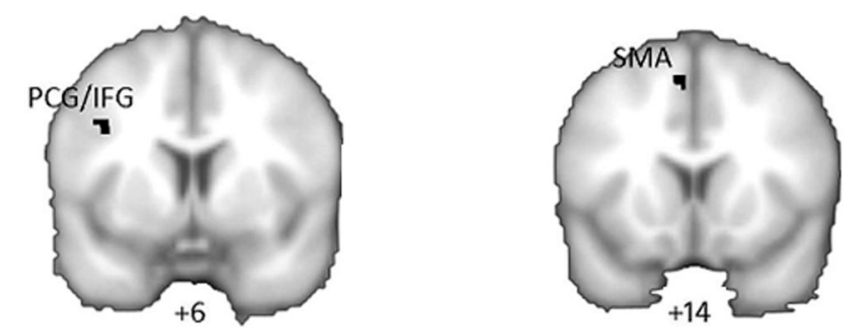

B.

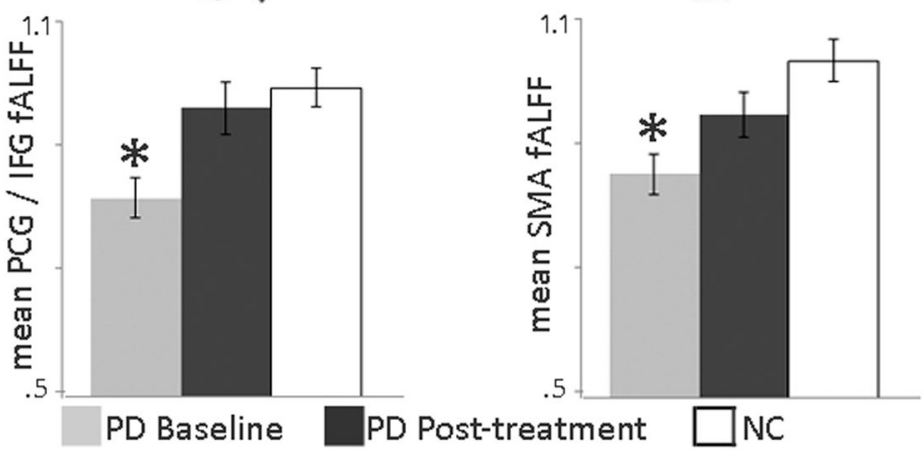

C.
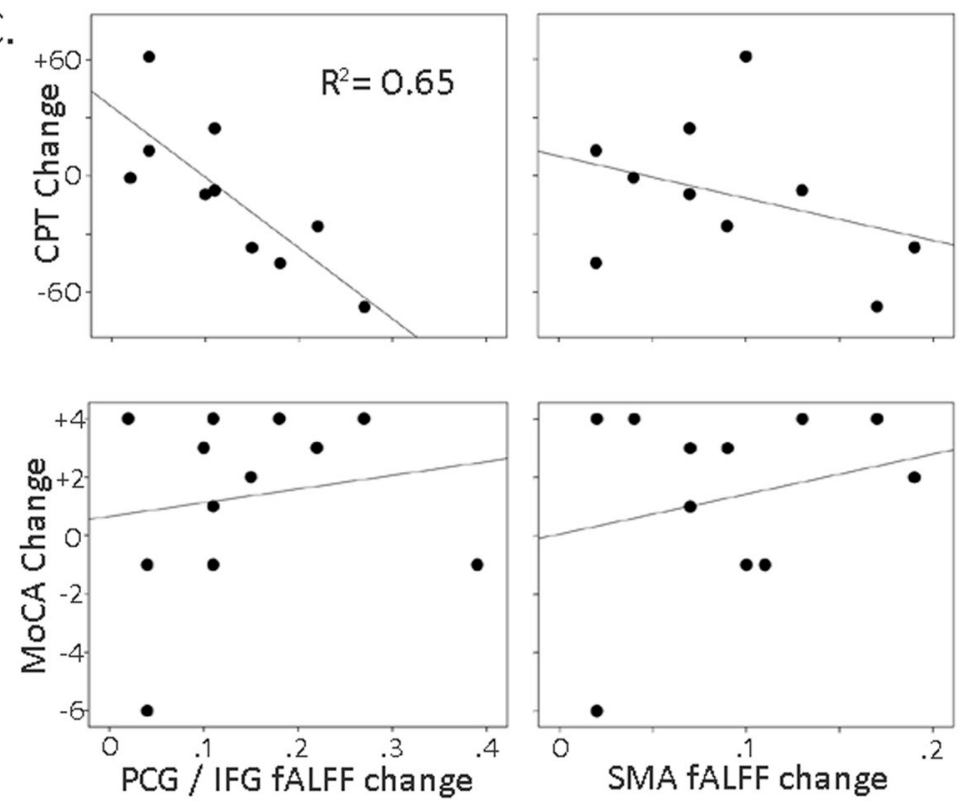

Figure 2.

A. Regions where PD patients showed greater fALFF at post-treatment compared to baseline. B. Bar graphs depict mean fALLF*100 with standard errors bars for each cluster by group. At baseline, the PD patients differed in mean fALLF in each cluster from post-treatment and from the NCs; the PD patients post-treatment did not differ from controls. C. The increase in local functional activity (mean falff*100) in the left precentral/inferior frontal gyri (PCG/IFG) cluster correlates significantly with improvement on the Continuous Performance Test (CPT). No other correlations with cognitive change scores were significant, including the correlation between this cluster and change on the Montreal Cognitive Assessment (MoCA) or the correlations between improvement in the left supplementary motor (SMA) cluster and change on the CPT or MoCA. Negative scores on the CPT Change indicate faster median reaction times (milliseconds) after treatment, and positive scores on the MoCA Change indicate higher MoCA scores after treatment. 


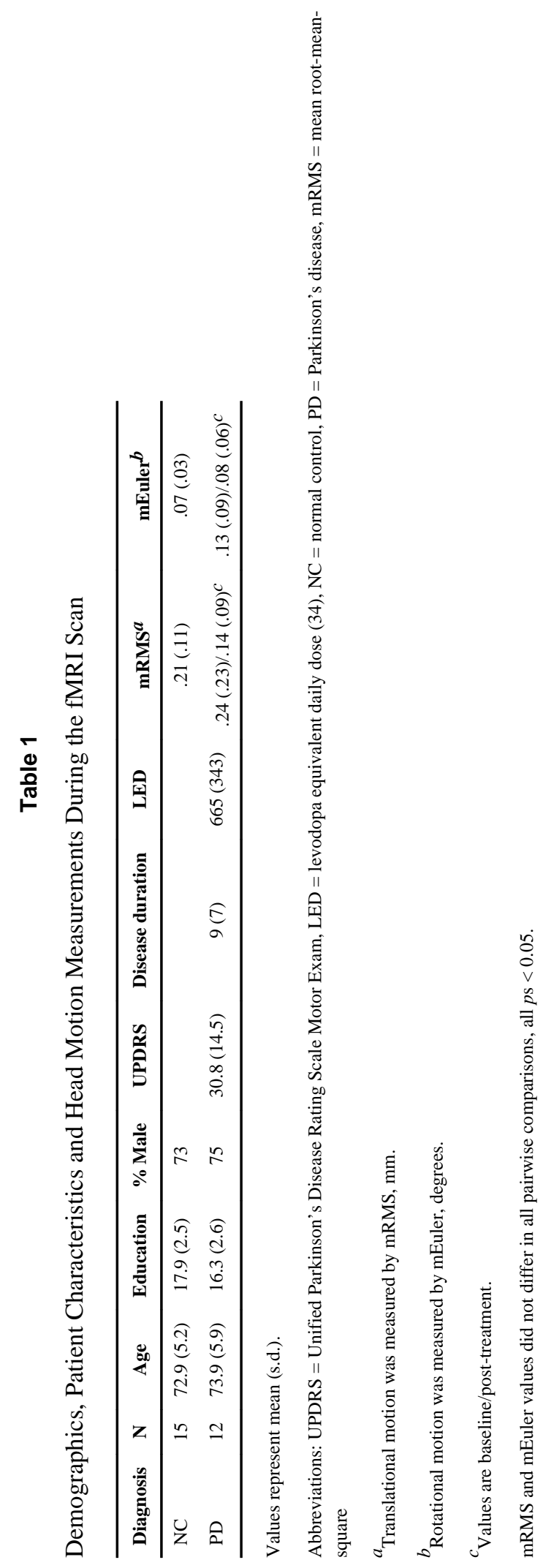

Mov Disord. Author manuscript; available in PMC 2014 September 01. 
